\title{
MULTIPLE PERIODIC SOLUTIONS FOR A CLASS OF SECOND-ORDER NONLINEAR NEUTRAL DELAY EQUATIONS
}

\author{
XIAO-BAO SHU AND YUAN-TONG XU
}

Received 14 October 2004; Accepted 12 December 2004

By means of a variational structure and $Z_{2}$-group index theory, we obtain multiple periodic solutions to a class of second-order nonlinear neutral delay equations of the form $x^{\prime \prime}(t-\tau)+\lambda(t) f(t, x(t), x(t-\tau), x(t-2 \tau))=x(t), \lambda(t)>0, \tau>0$.

Copyright (c) 2006 X.-B. Shu and Y.-T. Xu. This is an open access article distributed under the Creative Commons Attribution License, which permits unrestricted use, distribution, and reproduction in any medium, provided the original work is properly cited.

\section{Introduction}

Recently, the existence and multiplicity of periodic solutions for second-order neutral delay equations have received a good deal of attention (see, e.g., [3, 4, 7]). In [4], Wang and Yan studied the second-order neutral delay equation

$$
[x(t)+c x(t-\tau)]^{\prime \prime}+g(t, x(t-\sigma))=p(t),
$$

where $\tau, \sigma$, and $c$ are real constants with $\tau \geq 0, \sigma \geq 0,|c|<1, g(t, x)$ is a $T$-periodic function for $t>0$ and, for an arbitrary bounded domain $D \subset R, g(t, x)$ is a Lipschitzfunction on $[0, T] \times D$. Moreover, $p \in C(R, R), p(t+T)=p(t)$ and $\int_{0}^{T} p(t) d t=0$. They obtained sufficient conditions which guarantee the existence of at least one $T$-periodic solution for the above system.

However, for the existence of periodic solutions of functional differential equations, previous authors have used, mainly, fixed point theory, coincidence degree theory, Fourier analysis, and so forth. They have rarely used critical point theory. In $[5,6]$, the authors obtained multiple periodic solutions for a class of retarded differential equations by means of critical point theory and $Z_{p}$-group index theory. These results were obtained by reducing retarded differential equations to related ordinary differential equations.

The purpose of this paper is to establish a variational framework with delayed variables for a class of neutral differential equations. Unlike the papers $[5,6]$, our approach enables us to obtain by critical point theory and $Z_{2}$-group index theory the existence of 
2 Periodic solutions of neutral delay equations

nontrivial periodic solutions to such equations without reducing them to ordinary differential equations. To this end, we give below some preliminary material about $Z_{2}$-group index theory and critical points.

In what follows, $E$ is a real Banach space with norm $\|\cdot\|$.

Definition 1.1. A "critical point" of $f \in C^{1}(E, R)$ is a point $x \in E$ for which $f^{\prime}(x)=0$. A "critical value" of $f$ is a number $c$ such that $f(x)=c$ for some critical point $x$. The set $K=\left\{x \in E \mid f^{\prime}(x)=0\right\}$ is the "critical set" of $f$. We denote by $K_{c}$ the set $\left\{x \in E \mid f^{\prime}(x)=\right.$ $0, f(x)=c\}$. The "critical level" set $f_{c}$ of $f$ is defined by $f_{c}=\{x \in E \mid f(x) \leq c\}$.

Definition 1.2. Let $f \in C^{1}(E, R)$. We say that $f$ satisfies the "Palais-Smale" condition if every sequence $\left\{x_{n}\right\} \subset E$ such that $\left\{f\left(x_{n}\right)\right\}$ is bounded and $f^{\prime}\left(x_{n}\right) \rightarrow \theta$ as $n \rightarrow \infty$ has a convergent subsequence.

We say that a closed symmetric set $A \subset E$ satisfies property $\mathscr{P}$ if, for some $n \in Z^{+}$, there exists an odd continuous function $\varphi: A \rightarrow R^{n} \backslash\{\theta\}$. Let $N_{A} \subset Z$ be defined as follows: $n \in N_{A}$ if and only if $A$ satisfies property $\mathscr{P}$ with this $n$.

Definition 1.3. Let $E$ be real Banach space, and $\sum=\{A \mid A \subset E \backslash\{\theta\}$ a closed, symmetric set $\}$. Define $\gamma: \sum \rightarrow Z^{+} \bigcup\{+\infty\}$ as follows:

$$
\gamma(A)= \begin{cases}\min N_{A} & \text { if } N_{A} \neq \varnothing, \\ 0 & \text { if } A=\varnothing, \\ +\infty & \text { if } A \neq \varnothing, \text { but } N_{A}=\varnothing .\end{cases}
$$

We say that " $\gamma$ is the genus of $\sum$." We let $i_{1}(f)=\lim _{a \rightarrow-0} \gamma\left(f_{a}\right)$ and $i_{2}(f)=\lim _{a \rightarrow-\infty} \gamma\left(f_{a}\right)$. Lemma 1.4 (Chang [1]). Let $f \in C^{1}(E, R)$ be an even functional which satisfies the PalaisSmale condition and $f(\theta)=0$. Then

$\left(\mathrm{F}_{1}\right)$ if there exists an $m$-dimensional subspace $X$ of $E$ and $\rho>0$ such that

$$
\sup _{x \in X \cap S_{\rho}} f(x)<0,
$$

then we have $i_{1}(f) \geq m$;

$\left(\mathrm{F}_{2}\right)$ if there exists a $j$-dimensional subspace $\tilde{X}$ of $E$ such that

$$
\inf _{x \in \tilde{X}^{\perp}} f(x)>-\infty
$$

we have $i_{2}(f) \leq j$.

If $m \geq j$ and $\left(F_{1}\right),\left(F_{2}\right)$ hold, then $f$ has at least $2(m-j)$ distinct critical points.

In this paper, we use Lemma 1.4 to show the existence of multiple periodic solutions of the following second-order neutral delay equations:

$$
x^{\prime \prime}(t-\tau)+\lambda(t) f(t, x(t), x(t-\tau), x(t-2 \tau))=x(t-\tau), \quad \lambda(t)>0, \tau>0 .
$$

Our basic assumptions are the following:

$\left(\mathrm{A}_{1}\right) f\left(t, x_{1}, x_{2}, x_{3}\right) \in C\left(R^{4}, R\right)$, and $\partial f\left(t, x_{1}, x_{2}, x_{3}\right) / \partial t \neq 0$; 
$\left(\mathrm{A}_{2}\right)$ there exists a continuously differentiable function $F \in C^{1}\left(R^{3}, R\right)$ with such that

$$
F_{u_{2}}^{\prime}\left(t, u_{1}, u_{2}\right)+F_{u_{2}}^{\prime}\left(t, u_{2}, u_{3}\right)=f\left(t, u_{1}, u_{2}, u_{3}\right)
$$

(A $\left.\mathrm{A}_{3}\right) F\left(t+\tau, u_{1}, u_{2}\right)=F\left(t, u_{1}, u_{2}\right)$ for all $u_{1}, u_{2} \in R$, and $\lambda(t)$ is $\tau$-periodic in $t$.

$\left(\mathrm{A}_{4}\right) F$ satisfies: $F\left(t,-u_{1},-u_{2}\right)=F\left(t, u_{1}, u_{2}\right)$, and

$$
f\left(t,-u_{1},-u_{2},-u_{3}\right)=-f\left(t, u_{1}, u_{2}, u_{3}\right)
$$

By assumption $\left(\mathrm{A}_{2}\right)$, we have

$$
F_{u_{1}}^{\prime}(t, x(t-\tau), x(t-2 \tau))+F_{u_{2}}^{\prime}(t, x(t), x(t-\tau))=f(t, x(t), x(t-\tau), x(t-2 \tau)) .
$$

Thus, under assumptions $\left(A_{1}\right)-\left(A_{4}\right)$, we only need to study the following equation:

$$
x^{\prime \prime}(t-\tau)-x(t-\tau)+\lambda(t)\left[F_{u_{1}}(t, x(t-\tau), x(t-2 \tau))+F_{u_{2}}(t, x(t), x(t-\tau))\right]=0 .
$$

\section{Variational structure}

Fix $\gamma>0, \tau>0$, and consider

$H_{2 \gamma \tau}^{1}=\left\{x \in L^{2}[0,2 \gamma \tau] \mid x(t)\right.$ is a continuously differentiable $2 \gamma \tau$-periodic function in $\left.t\right\}$.

It is obvious that $H_{2 \gamma \tau}^{1}$ is a Sobolev space with inner product and norm defined by

$$
\begin{gathered}
\langle x, y\rangle_{H_{2 \gamma \tau}^{1}}=\int_{0}^{2 \gamma \tau}\left[x(t) y(t)+x^{\prime}(t) y^{\prime}(t)\right] d t \\
\|x\|_{H_{2 \gamma \tau}^{1}}=\left|\int_{0}^{2 \gamma \tau}\left[|x(t)|^{2}+\left|x^{\prime}(t)\right|^{2}\right] d t\right|^{1 / 2}, \quad \forall x, y \in H_{2 \gamma \tau}^{1} .
\end{gathered}
$$

Moreover, $x \in H_{2 \gamma \tau}^{1}$ can be expressed as follows;

$$
x(t)=a_{0}+\sum_{k=1}^{\infty}\left(a_{k} \cos \frac{k \pi}{\gamma \tau} t+b_{k} \sin \frac{k \pi}{\gamma \tau} t\right) .
$$

Let us consider the functional $I(x)$ defined on $H_{2 \gamma \tau}^{1}$ as follows:

$$
I(x)=\int_{0}^{2 \gamma \tau}\left[\frac{1}{2}\left(\left|x^{\prime}(t)\right|^{2}+|x(t)|^{2}\right)-\lambda(t) F(t, x(t), x(t-\tau))\right] d t .
$$


4 Periodic solutions of neutral delay equations

For all $x, y \in H_{2 \gamma \tau}^{1}$ and $\varepsilon>0$, we know that

$$
\begin{aligned}
I(x+\varepsilon y)=I(x)+\varepsilon \int_{0}^{2 \gamma \tau}[x(t) y(t) & +x^{\prime}(t) y^{\prime}(t) \\
& -\lambda(t)\left(F_{u_{1}}^{\prime}(t, x(t)+\varepsilon \theta(t) y(t), x(t-\tau)) y(t)\right. \\
& \left.\left.+F_{u_{2}}^{\prime}(t, x(t), x(t-\tau)+\varepsilon \theta(t) y(t-\tau)) y(t-\tau)\right)\right] d t \\
& +\frac{\varepsilon^{2}}{2} \int_{0}^{2 \gamma \tau}\left[y^{2}(t)+\left|y^{\prime}(t)\right|^{2}\right] d t \quad \forall x, y \in H_{2 \gamma \tau}^{1}, 0<\theta(t)<1 .
\end{aligned}
$$

It is easy to see that

$$
\begin{gathered}
\left\langle I^{\prime}(x), y\right\rangle=\int_{0}^{2 \gamma \tau}\left[x^{\prime}(t) y^{\prime}(t)+x(t) y(t)-\lambda(t) F_{u_{1}}^{\prime}(t, x(t), x(t-\tau)) y(t)\right. \\
\left.-\lambda(t) F_{u_{2}}^{\prime}(t, x(t), x(t-\tau)) y(t-\tau)\right] d t .
\end{gathered}
$$

By the periodicity of $F\left(t, u_{1}, u_{2}\right), \lambda(t), x(t), x(t-\tau)$, and $y(t)$, we get

$$
\begin{aligned}
\int_{0}^{2 \gamma \tau} \lambda(t) F_{u_{2}}^{\prime}(t, x(t), x(t-\tau)) y(t-\tau) d t & =\int_{-\tau}^{(2 \gamma-1) \tau} \lambda(t+\tau) F_{u_{2}}^{\prime}(t+\tau, x(t+\tau), x(t)) y(t) d t \\
& =\int_{0}^{2 \gamma \tau} \lambda(t) F_{u_{2}}^{\prime}(t, x(t+\tau), x(t)) y(t) d t .
\end{aligned}
$$

Hence,

$$
\begin{aligned}
\left\langle I^{\prime}(x), y\right\rangle=\int_{0}^{2 \gamma \tau}[ & -x^{\prime \prime}(t)+x(t)-\lambda(t)\left(F_{u_{1}}^{\prime}(t, x(t), x(t-\tau))\right. \\
& \left.\left.+F_{u_{2}}^{\prime}(t, x(t+\tau), x(t))\right)\right] y(t) d t
\end{aligned}
$$

Therefore, the Euler equation corresponding to the functional $I(x)$ is

$$
x^{\prime \prime}(t)-x(t)+\lambda(t)\left[F_{u_{1}}^{\prime}(t, x(t), x(t-\tau))+F_{u_{2}}^{\prime}(t, x(t+\tau), x(t))\right]=0 .
$$

It is easy to see that (2.9) is equivalent to (1.9). Thus, system (1.9) is the Euler equation of the functional $I(x)$. It follows that it is possible to obtain $2 \gamma \tau$-periodic solutions of system (1.5) by seeking critical points of the functional $I(x)$.

\section{Main results}

THeOREm 3.1. Let assumptions $\left(A_{1}\right)-\left(A_{4}\right)$ be satisfied and assume, further, that the function $F\left(t, u_{1}, u_{2}\right)$ satisfies the following:

$\left(\mathrm{C}_{1}\right) F(t, 0,0)=0, \forall t \in[0, \tau]$

$\left(\mathrm{C}_{2}\right)$

$$
\lim _{|u| \rightarrow 0} \frac{F\left(t, u_{1}, u_{2}\right)}{|u|^{2}}=1,
$$

where $|u|=\sqrt{u_{1}^{2}+u_{2}^{2}}$; 
$\left(\mathrm{C}_{3}\right)$ there exists $\alpha>0$ such that $F\left(t, u_{1}, u_{2}\right)<0$ whenever $u_{1}^{2}+u_{2}^{2}>\alpha, t \in[0, \tau]$.

Let $m=\min _{t \in[0, \tau]} \lambda(t)>0$. Then, for

$$
m>\frac{n^{2}\left(\pi^{2}+\gamma^{2} \tau^{2}\right)}{4 \gamma \tau^{2}},
$$

problem (1.5) has at least $2 n$ nontrivial $2 \gamma \tau$-periodic solutions.

It is not difficult to see that $x(t)$ is a solution of system (1.5), then $-x(t)$ is also a solution of system (1.5) by assumption $\left(\mathrm{A}_{4}\right)$, that is, the solutions of system (1.5) is a set that symmetric with respect to the origin in $H_{2 \gamma \tau}^{1}$. On the other hand, if we let $\eta(t, x)=$ $F(t, x(t), x(t-\tau))$, it is easy to see that $\eta(t, x)$ is an even function in $x$, so $I(x)$ an even function in $x$ and we can show that Theorem 3.1 holds by Lemma 1.4.

In order to exploit Lemma 1.4 to find the critical points of function $I(x)$ in $(2.4)$, one need to verify all the assumptions. First of all, we point out that the functional $I(\cdot)$ defined on $H_{2 \gamma \tau}^{1}$ satisfies the Palais-Smale condition, that is, we have the following lemma.

Lemma 3.2. Under assumptions $\left(A_{1}\right)-\left(A_{3}\right)$ and the conditions $\left(C_{1}\right)-\left(C_{3}\right), I(u)$ satisfies the P.S. condition.

Proof. Let $\left\{u_{n}\right\} \subset H_{2 \gamma \tau}^{1}$ and the constants $c_{1}, c_{2}$ satisfy

$$
\begin{aligned}
c_{1} & \leq I\left(u_{n}\right) \leq c_{2}, \\
I^{\prime}\left(u_{n}\right) & \longrightarrow 0 \quad(n \longrightarrow \infty) .
\end{aligned}
$$

The above equality is equivalent to

$$
\begin{aligned}
c_{1} \leq & \int_{0}^{2 \gamma \tau}\left[\frac{1}{2}\left(\left|u_{n}^{\prime}(t)\right|^{2}+\left|u_{n}(t)\right|^{2}\right)-\lambda(t) F\left(t, u_{n}(t), u_{n}(t-\tau)\right)\right] d t \leq c_{2}, \\
\sup \mid & \int_{0}^{2 \gamma \tau}\left[u_{n}^{\prime}(t) v^{\prime}(t)+u_{n}(t) v(t)\right] d t-\int_{0}^{2 \gamma \tau} \lambda(t) F_{u_{1}}^{\prime}\left(t, u_{n}(t), u_{n}(t-\tau)\right) v(t) d t \\
& -\int_{0}^{2 \gamma \tau} \lambda(t) F_{u_{2}}^{\prime}\left(t, u_{n}(t+\tau), u_{n}(t)\right) v(t) d t \mid \longrightarrow 0 \quad(n \longrightarrow \infty),
\end{aligned}
$$

where $v(t) \in H_{2 \gamma \tau}^{1},\|v\|=1$. Inequality (3.6) is equivalent to

$$
\begin{aligned}
\sup & \mid \int_{0}^{2 \gamma \tau}\left[u_{n}^{\prime}(t) v^{\prime}(t)+u_{n}(t) v(t)\right] d t-\int_{0}^{2 \gamma \tau} \lambda(t) F_{u_{1}}^{\prime}\left(t, u_{n}(t), u_{n}(t-\tau)\right) v(t) d t \\
& -\int_{0}^{2 \gamma \tau} \lambda(t) F_{u_{2}}^{\prime}\left(t, u_{n}(t), u_{n}(t-\tau)\right) v(t-\tau) d t \mid \longrightarrow 0 \quad(n \longrightarrow \infty)
\end{aligned}
$$

with $v(t) \in H_{2 \gamma \tau}^{1},\|v\|=1$.

Letting $z_{n}=I^{\prime}\left(u_{n}\right)$ and $\varepsilon_{n}=\left\|z_{n}\right\|$, we have $\varepsilon_{n} \rightarrow 0$ as $n \rightarrow \infty$.

Replacing $v$ by $u_{n}$ in (3.7), we have

$$
\begin{aligned}
\left\|u_{n}\right\|_{H_{2 \gamma \tau}^{1}}^{2}=\int_{0}^{2 \gamma \tau}[ & \lambda(t) F_{u_{1}}^{\prime}\left(t, u_{n}(t), u_{n}(t-\tau)\right) u_{n}(t) \\
& \left.+\lambda(t) F_{u_{2}}^{\prime}\left(t, u_{n}(t), u_{n}(t-\tau)\right) u_{n}(t-\tau)\right] d t+\left\langle z_{n}, u_{n}\right\rangle .
\end{aligned}
$$


6 Periodic solutions of neutral delay equations

By condition $\left(\mathrm{C}_{3}\right)$, we know that $F\left(t, u_{n}(t), u_{n}(t-\tau)\right)$ has an upper bound. Thus, by

$$
\max F\left(t, u_{n}(t), u_{n}(t-\tau)\right)=\max _{\left(t, u_{1}, u_{2}\right) \in[0, \tau] \times[-\alpha, \alpha] \times[-\alpha, \alpha]} F\left(t, u_{1}, u_{2}\right)=R>0,
$$

we get that $\int_{0}^{2 \gamma \tau} F\left(t, u_{n}(t), u_{n}(t-\tau)\right) d t \leq 2 \gamma \tau R$. Let $M=2 \gamma \tau R, Q=\max _{t \in[0, \tau]} \lambda(t)>0$, then

$$
\begin{aligned}
I\left(u_{n}\right) & =\int_{0}^{2 \gamma \tau}\left[\frac{1}{2}\left(\left|u_{n}^{\prime}(t)\right|^{2}+\left|u_{n}(t)\right|^{2}\right)-\lambda(t) F\left(t, u_{n}(t), u_{n}(t-\tau)\right)\right] d t \\
& \geq \frac{1}{2}\left\|u_{n}\right\|_{H_{2 \gamma \tau}^{1}}^{2}-Q M .
\end{aligned}
$$

By (3.5) and (3.10), it is easy to see

$$
\left\|u_{n}\right\|_{H_{2 \gamma \tau}^{1}} \leq \sqrt{2 Q M+2 c_{2}}
$$

that is, $\left\|u_{n}\right\|_{H_{2 y \tau}^{1}}$ is bounded.

Since $H_{2 \gamma \tau}^{1}$ is the Hilbert space of all continuously differentiable $2 \gamma \tau$-periodic functions and for a continuously $2 \gamma \tau$-periodic functions convergence sequences $\left\{x_{n}\right\}$ converges to a $2 \gamma \tau$-periodic function, it is not difficult to proof that conjugate space of $H_{2 \gamma \tau}^{1}$ is

$$
\begin{aligned}
H_{2 \gamma \tau}^{1 *}=\{ & x(t) \in L^{2}[0,2 \gamma \tau] \mid x(t) \text { is continuously } \\
& \text { differentiable } 2 \gamma \tau \text {-periodic function in } t\} .
\end{aligned}
$$

Since $H_{2 \gamma \tau}^{1}$ is a reflexive Banach space, that there exists a subsequence of $\left\{u_{n}\right\}$ which is weakly convergent in $H_{2 \gamma \tau}^{1}$. We denote, again, by $\left\{u_{n}\right\}$ and suppose that $u_{n} \rightarrow u_{0}$ in $H_{2 \gamma \tau}^{1}$ as $n \rightarrow \infty$.

By (3.8) and the boundedness of $\left\|u_{n}\right\|$, we get

$$
\begin{aligned}
\left\|u_{n}\right\|_{H_{2 \gamma \tau}^{1}}^{2} & -\int_{0}^{2 \gamma \tau} \lambda(t) F_{u_{1}}^{\prime}\left(t, u_{n}(t), u_{n}(t-\tau)\right) u_{n}(t) d t \\
& -\int_{0}^{2 \gamma \tau} \lambda(t) F_{u_{2}}^{\prime}\left(t, u_{n}(t), u_{n}(t-\tau)\right) u_{n}(t-\tau) d t \rightarrow 0 \quad(n \longrightarrow \infty) .
\end{aligned}
$$

On the other hand, the weak convergence of $\left\{u_{n}\right\}$ of $H_{2 \gamma \tau}^{1}$ implies the uniform convergence of $\left\{u_{n}\right\}$ in $C([0,1], R)$ (see [2]). Hence,

$$
\begin{aligned}
\left\|u_{n}\right\|_{H_{2 \gamma \tau}^{1}}^{2} \longrightarrow & \int_{0}^{2 \gamma \tau} \lambda(t) F_{u_{1}}^{\prime}\left(t, u_{n}(t), u_{n}(t-\tau)\right) u_{n}(t) d t \\
& +\int_{0}^{2 \gamma \tau} \lambda(t) F_{u_{2}}^{\prime}\left(t, u_{n}(t), u_{n}(t-\tau)\right) u_{n}(t-\tau) d t \quad(n \longrightarrow \infty) .
\end{aligned}
$$

This means that $\left\|u_{n}\right\|$ is convergent in $H_{2 \gamma \tau}^{1}$, that is, the function $I$ satisfy P.S. condition. 
Lemma 3.3. Under assumptions $\left(A_{1}\right)-\left(A_{4}\right)$ and conditions $\left(C_{1}\right)-\left(C_{3}\right)$, there exists an $n$ dimensional subspace $E_{n}$ of $H_{2 \gamma \tau}^{1}$ and $\rho>0$ such that

$$
\sup _{x \in E_{n} \cap S_{\rho}} I(x)<0,
$$

that is, we have $i_{1}(f) \geq n$.

Proof. Let $\beta_{k}(t)=(\gamma \tau / k \pi) \cos ,(k \pi / \gamma \tau) t, k=1,2,3, \ldots, n, \ldots$, then

$$
\int_{0}^{2 \gamma \tau}\left|\beta_{k}(t)\right|^{2} d t=\frac{\gamma^{2} \tau^{2}}{k^{2} \pi^{2}} \gamma \tau, \quad \int_{0}^{2 \gamma \tau}\left|\beta_{k}^{\prime}(t)\right|^{2} d t=\gamma \tau .
$$

We define the $n$-dimensional linear space $E_{n}$ as follows:

$$
E_{n}=\operatorname{span}\left\{\beta_{1}(t), \beta_{2}(t), \ldots, \beta_{n}(t)\right\} .
$$

Obviously, $E_{n}$ is symmetric. Suppose that $\rho>0$. Then

$$
E_{n} \bigcap S_{\rho}=\left\{\sum_{k=0}^{n} b_{k} \beta_{k} \mid \sum_{k=0}^{n} b_{k}^{2} \gamma \tau\left(1+\frac{\gamma^{2} \tau^{2}}{k^{2} \pi^{2}}\right)=\rho^{2}\right\} .
$$

On the other hand, we may choose $\varepsilon>0$ such that $0<\varepsilon<\left(m n^{2} \pi^{2} / \gamma^{2} \tau^{2}\right)\left(2 \gamma^{2} \tau^{2} / n^{2}-\left(\pi^{2}+\right.\right.$ $\left.\gamma^{2} \tau^{2}\right) / m$ ). Then, by condition $\left(\mathrm{F}_{2}\right)$, we know that there exists $\delta>0$ such that

$$
\begin{aligned}
\lambda(t) F(t, x(t), x(t-\tau)) & \geq(\lambda(t)-\varepsilon)\left[|x(t)|^{2}+|x(t-\tau)|^{2}\right] \\
& \geq(m-\varepsilon)\left[|x(t)|^{2}+|x(t-\tau)|^{2}\right] \quad \forall t \in[0,2 \gamma \tau],
\end{aligned}
$$

whenever $\left(\left\|x_{n}(t)\right\|_{C}^{2}+\| x\left(t-\tau \|_{C}^{2}\right) \leq \delta\right.$, where $\left\|x_{n}(t)\right\|_{C}^{2}=\max _{0 \leq t \leq 2 \gamma \tau}|x(t)|$. Thus, when we choose $\rho=\delta$, we get, by the periodicity of $x(t), x(t-\tau)$,

$$
\begin{aligned}
I(x) & =\int_{0}^{2 \gamma \tau}\left[\frac{1}{2}\left(\left|x^{\prime}(t)\right|^{2}+|x(t)|^{2}\right)-\lambda(t) F(t, x(t), x(t-\tau))\right] d t \\
& \leq \frac{1}{2} \sum_{k=0}^{n} \gamma \tau b_{k}^{2}\left(1+\frac{\gamma^{2} \tau^{2}}{k^{2} \pi^{2}}\right)-(m-\varepsilon) \int_{0}^{2 \gamma \tau}\left[|x(t)|^{2}+|x(t-\tau)|^{2}\right] d t \\
& \leq \frac{1}{2} \sum_{k=0}^{n} \gamma \tau b_{k}^{2}\left(1+\frac{\gamma^{2} \tau^{2}}{k^{2} \pi^{2}}\right)-2(m-\varepsilon) \int_{0}^{2 \gamma \tau}|x(t)|^{2} d t \\
& \leq \frac{1}{2} \sum_{k=0}^{n} \gamma \tau b_{k}^{2}\left(1+\frac{\gamma^{2} \tau^{2}}{k^{2} \pi^{2}}\right)-2(m-\varepsilon) \sum_{k=0}^{n} \gamma \tau b_{k}^{2} \frac{\gamma^{2} \tau^{2}}{k^{2} \pi^{2}} \\
& \leq \frac{1}{2} \sum_{k=0}^{n} \gamma \tau b_{k}^{2}\left(1+\frac{\gamma^{2} \tau^{2}}{\pi^{2}}\right)-2(m-\varepsilon) \sum_{k=0}^{n} \gamma \tau b_{k}^{2} \frac{\gamma^{2} \tau^{2}}{n^{2} \pi^{2}} \\
& \leq \frac{m \gamma \tau}{2 \pi^{2}}\left(\frac{\pi^{2}+\gamma^{2} \tau^{2}}{m}-\frac{4 \gamma^{2} \tau^{2}}{n^{2}}+\varepsilon \frac{\gamma^{2} \tau^{2}}{m n^{2} \pi^{2}}\right)<0 \quad \forall x \in E_{n} \bigcap S_{\rho},
\end{aligned}
$$

that is, Lemma 3.3 holds true. 
8 Periodic solutions of neutral delay equations

Remark 3.4. The above equality makes use of

$$
m>\frac{n^{2}\left(\pi^{2}+\gamma^{2} \tau^{2}\right)}{4 \gamma \tau^{2}}, \quad 0<\varepsilon<\frac{m n^{2} \pi^{2}}{\gamma^{2} \tau^{2}}\left(\frac{2 \gamma^{2} \tau^{2}}{n^{2}}-\frac{\pi^{2}+\gamma^{2} \tau^{2}}{m}\right) .
$$

From (3.10) we know that $I(x)$ has a lower bound, that is, $i_{2}(I)=0$. On the other hand, by condition $\left(\mathrm{C}_{1}\right)$, we get $I(\theta)=0$. So, by Lemmas 3.2 and 3.3, we obtain Theorem 3.1.

Example 3.5. Let

$$
F\left(t, u_{1}, u_{2}\right)=u_{1}^{2}+u_{2}^{2}-\left[1+\sin ^{2} \frac{\pi t}{\tau}\right]\left(u_{1}^{2}+u_{2}^{2}\right)^{2}
$$

Then

$$
\begin{aligned}
& F_{u_{1}}(t, x(t-\tau), x(t-2 \tau))+F_{u_{2}}(t, x(t), x(t-\tau)) \\
&= 4 x(t-\tau)-\left[1+\sin ^{2} \frac{\pi t}{\tau}\right]\left(4\left(x^{2}(t-\tau)+x^{2}(t-2 \tau)\right) x(t-\tau)\right. \\
&\left.+4\left(x^{2}(t)+x^{2}(t-\tau)\right) x(t-\tau)\right) \\
&= 4 x(t-\tau)-4\left[1+\sin ^{2} \frac{\pi t}{\tau}\right]\left(x^{2}(t)+2 x^{2}(t-\tau)+x^{2}(t-2 \tau)\right) x(t-\tau) .
\end{aligned}
$$

Let

$$
\begin{aligned}
& f_{1}(t, x(t), x(t-\tau), x(t-2 \tau)) \\
& \quad=4 x(t-\tau)-4\left[1+\sin ^{2} \frac{\pi t}{\tau}\right]\left(x^{2}(t)+2 x^{2}(t-\tau)+x^{2}(t-2 \tau)\right) x(t-\tau) .
\end{aligned}
$$

It is easy to see that $f_{1}\left(t,-u_{1},-u_{2},-u_{3}\right)=-f_{1}\left(t, u_{1}, u_{2}, u_{3}\right)$ and

$$
\lim _{|u| \rightarrow 0} \frac{F\left(t, u_{1}, u_{2}\right)}{|u|^{2}}=\lim _{|u| \rightarrow 0} \frac{u_{1}^{2}+u_{2}^{2}-\left[1+\sin ^{2}(\pi t / \tau)\right]\left(u_{1}^{2}+u_{2}^{2}\right)^{2}}{u_{1}^{2}+u_{2}^{2}}=1 .
$$

Since

$$
F\left(t, u_{1}, u_{2}\right)<0, \quad \forall t \in[0, \tau]
$$

whenever $u_{1}^{2}+u_{2}^{2}>1$, all the conditions of Theorem 3.1 hold true. By Theorem 3.1, we get that the problem

$$
x^{\prime \prime}(t-\tau)+\lambda(t) f_{1}(t, x(t), x(t-\tau), x(t-2 \tau))=x(t-\tau),
$$

with $\lambda(t)$ continuous and positive, has at least $2 n$ nontrivial $2 \gamma \tau$-periodic solutions when $m>n^{2}\left(\pi^{2}+\gamma^{2} \tau^{2}\right) / 4 \gamma \tau^{2}$. Where $m=\min _{t \in[0, \tau]} \lambda(t)$.

\section{Acknowledgment}

This project was supported by NNSF of China (10471155), the Foundation of the Guangdong Province Natural Science Committee (031608) and a specific Foundation for Ph.D. Specialities of Educational Department of China (20020558092). 


\section{References}

[1] K. C. Chang, Critical Point Theory and Its Applications, Xiandai Shuxue Congshu, Shanghai Kexue Jishu Chubanshe, Shanghai, 1986.

[2] J. Mawhin and M. Willem, Critical Point Theory and Hamiltonian Systems, Applied Mathematical Sciences, vol. 74, Springer, New York, 1989.

[3] G. Q. Wang, Periodic solutions to second-order neutral equations, Gaoxiao Yingyong Shuxue Xuebao. Applied Mathematics. A Journal of Chinese Universities. Series A (Chinese Edition) 8 (1993), no. 3, 251-254 (Chinese).

[4] G. Q. Wang and J. R. Yan, Existence of periodic solutions for second order nonlinear neutral delay equations, Acta Mathematica Sinica 47 (2004), no. 2, 379-384 (Chinese).

[5] Y.-T. Xu and Z.-M. Guo, Applications of a $Z_{p}$ index theory to periodic solutions for a class of functional differential equations, Journal of Mathematical Analysis and Applications 257 (2001), no. 1, 189-205.

[6] __ Applications of a geometrical index theory to functional differential equations, Acta Mathematica Sinica 44 (2001), no. 6, 1027-1036 (Chinese).

[7] Y. Zhang and Y. Zhang, Periodic solutions to second-order linear neutral differential equations with constant coefficients, Acta Mathematica Sinica. Shuxue Xuebao 33 (1990), no. 4, 517-520 (Chinese).

Xiao-Bao Shu: Department of Mathematics, Hunan University, Changsha, 410082, China E-mail address: sxb0221@163.com

Yuan-Tong Xu: Department of Mathematics, Sun Yat-sen (Zhongshan) University, Guangzhou 510275, China

E-mail address: xyt@zsu.edu.cn 


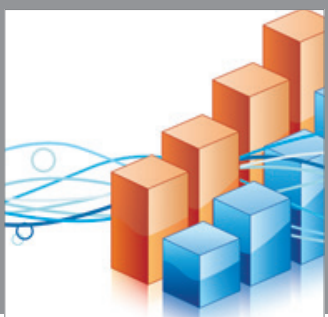

Advances in

Operations Research

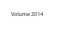

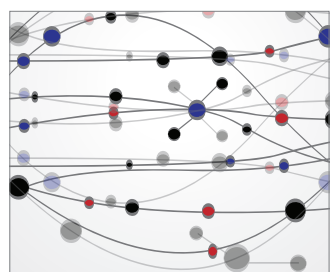

\section{The Scientific} World Journal
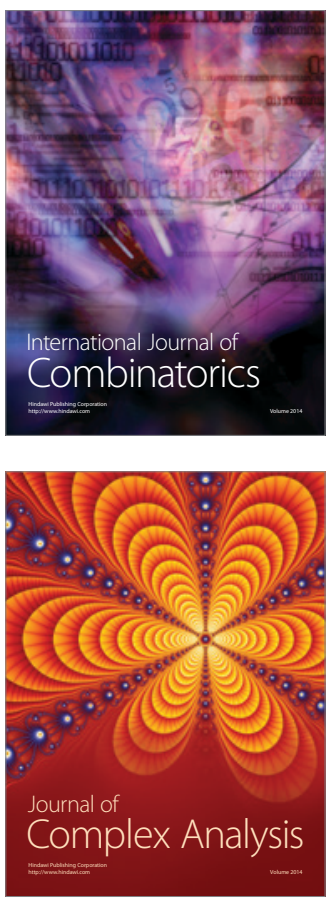

International Journal of

Mathematics and

Mathematical

Sciences
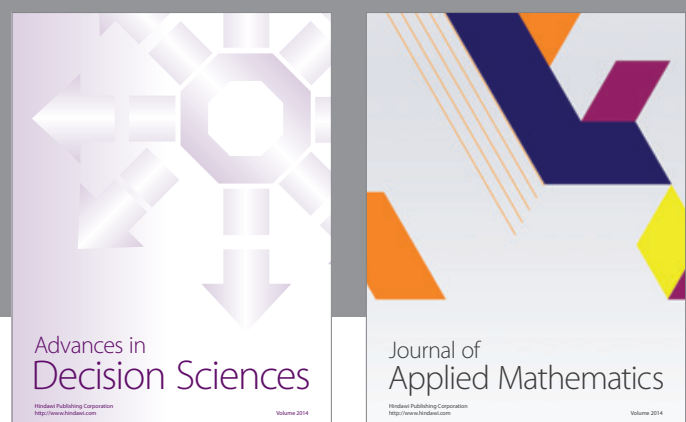

Journal of

Applied Mathematics
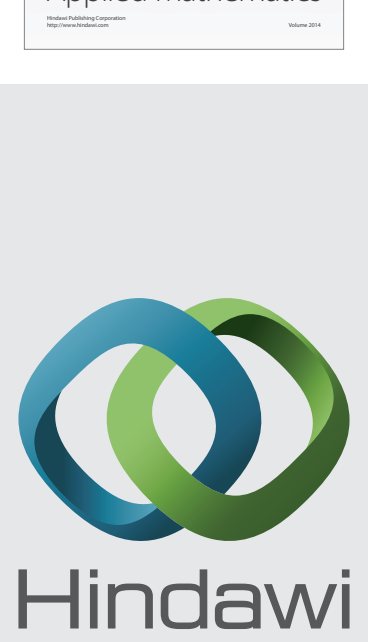

Submit your manuscripts at http://www.hindawi.com
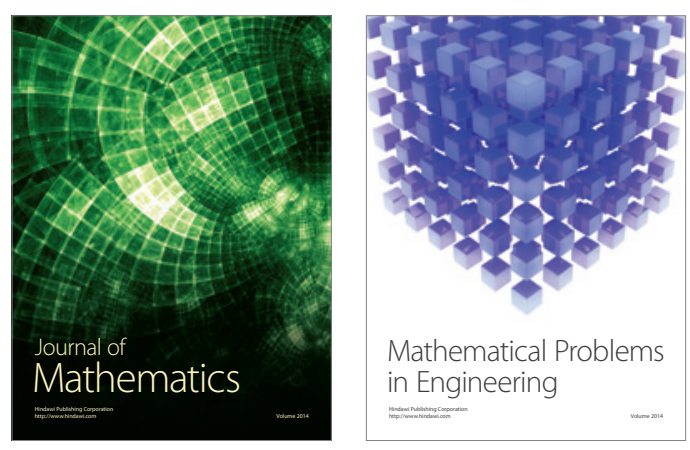

Mathematical Problems in Engineering
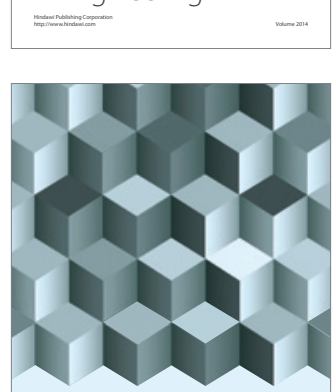

Journal of

Function Spaces
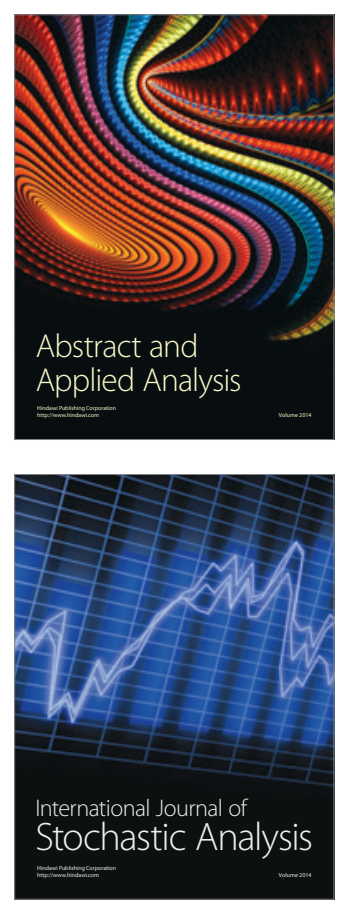

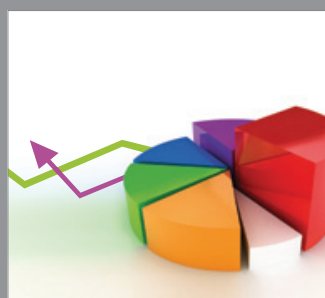

ournal of

Probability and Statistics

Promensencen
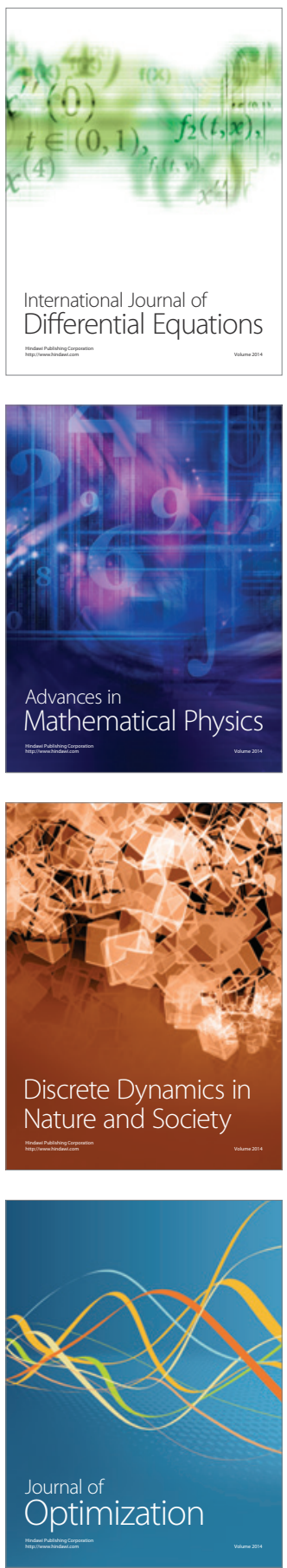